\title{
Movement of Autonomous Vehicles in Work Zone Using New Pavement Marking: A New Approach
}

\author{
Prashant Singh $^{1 *}$, Md Ahsanul Islam² \\ ${ }^{1}$ Graduate Research Assistant, Department of Civil \& Coastal Engineering, University of Florida, Gainesville, FL, USA \\ ${ }^{2}$ Associate Traffic Engineer, Parsons Corporation, East Hartford, Connecticut, USA \\ Email: ^Prashantsingh@ufl.edu, ahsancuet08@gmail.com
}

How to cite this paper: Singh, P. and Islam, Md.A. (2020) Movement of Autonomous Vehicles in Work Zone Using New Pavement Marking: A New Approach. Journal of Transportation Technologies, 10, 183-197. https://doi.org/10.4236/jtts.2020.103012

Received: April 5, 2020

Accepted: May 8, 2020

Published: May 11, 2020

Copyright $\odot 2020$ by author(s) and Scientific Research Publishing Inc. This work is licensed under the Creative Commons Attribution International License (CC BY 4.0).

http://creativecommons.org/licenses/by/4.0/

\begin{abstract}
The autonomous vehicles are the future of mobility across the globe and are expected to touch the lives of every person of all ages. But this comes with certain challenges regarding safety, reliability, cost, legal framework, regulations, etc. however, of all the concern safety and reliability are of utmost importance for researchers and engineers. The current research is focused on the movement of the autonomous vehicle in the work zone. The work zone is one of the most challenging areas for the autonomous vehicle to drive from. This is because the work zones are very dynamic, and all the construction activities are specific to the site condition and cannot always be predefined. The study provides a concept of how pavement marking can be used for smooth movement through the complicated work zone. In this study, various pavement marking signs have been designed as a concept considering the standard colors and striping width being used in the Manual of Uniform Traffic control device (MUTCD). The study assumes that the movement of the autonomous vehicle will not be exclusive and that it will move with the driver driven vehicle. It is expected that autonomous vehicles will require special pavement marking and signage for smooth movement through the work zone. These pavement marking and signage will eventually become part of standard Traffic Control Plans (TCP) and Maintenance of Traffic Plans (MOT). The research aims to study the current research being done in this area and technology being used for detecting various pavement markings and signages.
\end{abstract}

\section{Keywords}

Autonomous Vehicle Movement in Work Zone, Pavement Markings, MUTCD, Traffic Control Plans, Maintenance of Traffic Plans, Work Zone Safety 


\section{Introduction and Motivation}

Millions of people around the world start and end their day commuting in overcrowded public transit systems like buses and trains. Therefore, improving the quality of commute and accessibility is very critical to improving the life of the people. With the advent of smart cities concept across the globe, the data and digital technology work is improving the quality of life. More comprehensive, real-time data gives agencies the ability to understand how demand patterns are changing and respond with faster and lower-cost solutions. Apart from this, the effective use of real-time data and technology is providing people with more choices in decision making.

From a global perspective, mobility focus is both within the urban settlements and rural habitation. Smart city initiatives have put mobility at the forefront of all technology research. If we look at the key areas of focus on various mobility applications, the major ones are Real-time public transit information, Digital public transit payment, Autonomous vehicles (AVs), Predictive maintenance of transportation infrastructure, Intelligent traffic signals, Congestion pricing, Demand-based micro-transit Smart parking E-hailing (private and pooled), Car sharing, Bike sharing, integrated multimodal information Real-time road navigation, Parcel load pooling, and Smart parcel lockers. All these are directed towards providing people with better choices and make life and travel easier. The movement of (AVs) is the focus area of most research and is expected to transform the future of travel.

AVs or driverless vehicles use the latest technology using the GPS (Global Positioning System) navigation system, radar, LiDAR (Light Detection and Ranging), odometer, sensors to perceive and recognize the signal and traffic signs and on-board computers capable of advanced algorithms and machine [1] [2] [3].

There is no doubt that our roads are made for the human drivers and our policies and legislations are made to cater to this. With the advent of AVs, the construction experts and policymakers will need to work together to see how AVs can navigate through the work zone without limiting its ability to move in the construction work zone safely.

There are many areas of autonomous vehicle limitation. The movement of the autonomous vehicle in work zones remains a challenge to the researchers and automobile manufactures. The main reason is work zones are very dynamic and are always site specifics. The changes in traffic control plans are sometimes so frequent that it is very difficult to write a code of each and information. The major problem area in the movement of AVs in the work zone involves identification of the work zone, detecting the lane closure, detours, understanding the signage and interaction with the human drivers.

Based on various current researches and existing infrastructure that is available for the movement of autonomous vehicles. There is an agreement with the expert and technologist that the available infrastructure is for the vehicle driven by the human and that there is a need for the enhancement of the existing infrastructure to respond to the necessity of connected and autonomous vehicles. 
Various concern areas have been identified that may hamper the movement of the autonomous vehicle. The few of the major concern areas are:

- There is a concern for the autonomous vehicles to negotiate through the work zone being handled through flaggers. Since AVs is designed to scan the roadway signs but the use of flagger and understanding its movement direction is still an area of concern.

- Another area of concern is us of confusing pavement marking in the work zone. During traffic control plans of the roadway. The removal of striping and laying new striping can create a lot of confusion for AVs which tends to scan through the pavement marking to negotiate its trajectory safely.

- One more area of concern is changing the width of a travel lane in the work zone especially on the ramps where the right of way is limited and width of the travel way changes from 12 feet to 8 feet and sometimes even less. With the use of low-profile barrier wall or type ' $\mathrm{K}$ ' barrier wall on the side can cause $\mathrm{AVs}$ to react differently as the close proximity of AVs to road furniture can make sensors predict the potential hazard and can eventually stop the vehicle.

- There is a concern with the placement of road sign on-ramps and its interpretation.

- One more area of concern is the use of different pavement marking on the highway. With the idea of making roads safer efforts are being made to use different pavement marking so that they are safer in all driving conditions. This non-uniformity can cause AVs to behave differently.

- In a work zone area of complex nature where traffic control plan will change more frequently. It is not certain as to how AVs will react.

Based on the above area of concern for the movement of AVs the main objective of this paper is to study the movement of AVs in the work zone. This paper seeks to explore the various aspects of the movement of AVs in the construction work zone, use of technology and standardize the pavement marking and design of new signage for the work zone.

The paper discusses the following major aspects:

- Lane detection and departure warning technologies;

- The major impact of the autonomous vehicles work zone;

- Current research and analysis;

- Challenges in implementing autonomous vehicles in the work zone.

An attempt has been made in the paper to present a solution by designing a new set of pavement markings that can facilitate AVs movement through the work zone.

\section{Literature Review}

There has been a lot of research done to make the work zone as an intelligent system using connected vehicle technologies to reduce crashes. With the advent of autonomous vehicles, the work zones have become an important area of research for academician and engineers. This is mostly because the nature of the work zone is very dynamic and site-specific such that it very difficult to write 
code for everything especially when an autonomous vehicle is crossing it. Not much research work has been done regarding designing a pavement marking based system which can be used to safely move the autonomous vehicles through the dynamic work zone.

According to Fengxiang Qiao [4] the intelligent work zone system are developed combining the functions of detection, system monitoring, system communication, system analysis, data management, and dynamic information. A lot of work is being done in the area of lane marking detection using advanced detection systems.

Possibility of deployment of driverless vehicles introduced not only the possible improvement of safety due to the limitation of human errors but also the utilization of unoccupied cars. Michal [5] discusses the lane assisted system (LAS) in the Czech Republic. The utilization of pavement marking is an important aspect of the movement of the autonomous vehicle in the work zone. As one of the intelligent functionality, lane detection is the problem of locating lane boundaries [6]. Until present, various vision-based lane detection algorithm has been developed. They usually utilize different lane patterns (solid or dashed white painted lines, etc.). There has been significant development of research on machine vision for road vehicles [7] and the lane boundary detection has been active research over the last decade. It is expected that an automatic lane detector should be able to detect both straight and curved lane boundaries, the full range of the lane markings (single, double and solid or broken) and marking edge under a variety of type, lane structure, weather condition, shadows, puddle, stains, and highlight.

Of the many approaches for lane detection, only a few works deal with extraction and analysis of road marking. In [8] a lane marker extractor is presented, and the concatenation of these markings is used to estimate the course of the lane. The combination of road borders and road markers was used in [9] for lane detection. Another lane marker extractor is presented in [10]. Stephen Vacek [11] suggests an analysis of two different types of information to classify road markings. The first one is the type of lines e.g. solid or dashed and the second one is the arrow which is painted on the road. The four-step classification of lines starts with a sampling of the painted lines using scanlines. A scanline is a straight line orthogonal to the painted road marking and is represented in $3 \mathrm{D}$ world coordinates in the vehicle coordinate system. Other steps of classification are classification of scanlines, concatenation of scanlines and line type classification.

Alex Voronov [12] conducted a pre-study of equipping infrastructure with radar reflectors. They estimated that reflector should be installed in the lane marking, about 20 - $25 \mathrm{~m}$ apart with some kind of identification. A combi-reflector which can be equipped with a magnet for the in-vehicle electromagnet. Currently, vehicles are using a combination of technologies and sensor fusion which rely on visible light through cameras for detection and objects in the infrastructure for reflection like lane markings, snow poles, delineator posts, traffic barriers 
and guardrails, traffic signs, reflective pavement marking. Voronov [12] outlines several approaches to identify the reflectors, including changing radar cross-section, polarization, and encoding position in the distances between the reflectors, adding additional camera readable elements and putting several reflectors in a row laterally or longitudinally.

Narote [13] outlines various advances in lane detection and departure warning systems and lists the summary and detailed description of lane detection and departure warning systems [13]. Also concludes that the Hough transform is extensively used to detect lane marks, but it requires large computational time. Also, the problem of lane detection under complex environmental conditions involving shadow, dynamic illumination condition, dense fog, rainy condition, nigh time and bad condition of lane markers, remains an unsolved problem.

Danilo [14] discussed the problem of excessive processing time and false detection due to similarities in color and edges between traffic signs, channelizing lines, stop lines, crosswalks, arrows, etc. [14]. proposes a strategy to extract the lane marking information by taking into consideration its feature such as color, edge, and width, as well as vehicle speed. Using these parameters, the regions are computed by using a road geometric model. The study also concludes the following results: First, to introduce an automated method for extracting the region of interest-based on the relationship between the vehicle speed and the typical stopping distance within the image. As a result, the image is sectioned into three regions: the information above the horizon, a zero-collision risk, and a collision risk region. Second, to propose a real-time lane marking detection strategy combining edge and color features, which uses the probability that the extracted features define a lane marking, as well as a hierarchical fitting model. As a result, the method can detect lane markings with an accuracy of $96.84 \%$ at an average processing time of $28.30 \mathrm{~ms}$, for a speed range of $5-45 \mathrm{~km} / \mathrm{h}$. Similarly, the algorithm solves the problem given by traffic marking signal types such as channelizing lines, stop lines, crosswalks, and arrows. Third, to estimate the lane region and the vehicle heading angle by using the road geometry model and the lane centroid information.

Few studies have been done to study the impact of autonomous and connected vehicles on the work zone in the freeway. Michelle [15] used the integration of LiDAR (Light Detection and Ranging) generated geometric data with connected vehicle speed data to evaluate the impact of work zone geometry on traffic operations. Two case studies where geometric anomalies were identified are discussed in this paper. For this, a short segment with a narrow lane width of 10 - 10.5 feet and a merging taper that was about 200 feet shorter than recommended by the manual on uniform traffic control devices was used. To manage the work zone traffic control compliance and quality New York state department of transportation developed a construction and health safety program [16] which includes quality assurance program and inspection procedure [17]. The study shows how connected vehicle data can be used to identify work zone with sig- 
nificant congestion, and how LiDAR can be used to identify geometric conditions that deviate from design and are likely contributing to the work zone queuing. There are three aspects of LiDAR deployment [15]. First, all agencies apply highly reflective glass beads or elements to pavement marking to improve night visibility. Well, maintain retroreflective marking provides very high contrast in LiDAR point cloud and can be easily extracted via automated processes [15]. Second, environmental conditions need to be considered when using LiDAR. Unlike video data collection, LiDAR can be used at night. In one night time lane closure workers in reflective gear standing in front of floodlight were more visible in the LiDAR point cloud than could be visibly observed by the driver. However, LiDAR performance is significantly degraded when there is precipitation [15]. Third, the speed at which mobile LiDAR travels affects the density of the data point cloud, slower speed will produce denser point cloud but may be less safe on high-speed facilities, such as freeway. This study chooses $40 \mathrm{mph}$ as speed. The study concludes that the integration of LiDAR geometric data with the connected vehicle speed data enables the diagnosis of the non-conforming geometric condition in the work zone.

Andrew L. B. [18] Demonstrate that inexpensive expendable radio frequency identification (RFID) tags could be used to identify the spatial location along the pavement alignment of specific truckloads of hot mix asphalt (HMA) production. These tags are placed in the truckload as it leaves the production plant, passes through the paver, and are compacted into the finished mat. Cross-referencing these tags with Global Positioning System (GPS) latitude and longitude coordinates after construction allows spatial referencing of quality assurance (QA) material property data measured at the production plant, enabling linkage to other spatially referenced in-place test results and pavement management system (PMS) pavement performance data [18]. Usually, there are two types of RFID technology that was employed in this study. One is a conventional passive ultrahigh-frequency (UHF) RFID tags and the newer SAW RFID tags. The passive UHF RFID technology encodes a digital signature on a small microchip attached to a copper foil antenna-the RFID "tag". This passive tag receives energy from UHF radio waves transmitted by an RFID "reader"; the tag harvests this incoming radio frequency $(\mathrm{RF})$ energy to transmit its encoded digital signature back to the reader. These RFID tags, although small, can be read several yards away from the reader's antenna. The best part of SAW is that the SAW chip operates in a purely passive mode and does not require supplementary direct current (DC) power (i.e., battery). [18] concludes in the study that the field trials confirmed the very high survival rate of the encapsulated RFID tags. Read success rates varied significantly with tag size and less significantly on other details such as antenna configuration and vehicle speed. The field trials consistently demonstrated that post-construction read success rates of 60 to 80 percent or higher are achievable from a bumper-mounted antenna array, even on a vehicle moving at traffic speeds. 
[19] [20] and [21] says approximately 25 percent of the crashes in the study set is not traditional ROR crashes but crashes where the vehicle left the roadway at a gentle angle, then oversteered back onto the roadway, resulting in a loss of control and a subsequent crash either with another vehicle, a fixed object on the same or opposite side of the road, or due to overturning because of loss of lateral stability. It is presumed that ROR crashes can be stopped in autonomous vehicles with the implementation of standardized protocol both during and after construction.

\section{Analysis and Results}

AVs generally utilize LiDAR, radar, or both in addition to cameras, ultrasonic sensors, inertial measurement systems, detailed mapping and infrared technologies for their movement [22]. These act in three phases that can be compared to a human ability to sense, decide, and act in any given scenario. In phase one, all of these instruments collect data from the environment and are an augmented representation of the human's visual and cognitive system. In phase two, the instruments combine information and, via advanced computing and algorithms, form a plan of action much as the human brain operates cognitively. In the last phase, the AVs act or execute that plan, which may include a maneuver, a change in acceleration, or some other driving action [23].

Through various researches, efforts are being made for the detection of pavement marking in the work zone. However, there are various areas of autonomous limitations like visibility in adverse weather, navigating work zones or temporary traffic patterns and behavior around emergency vehicles. Though adverse weather conditions can substantially reduce the performance of sensors, radars, and LiDAR. This may compromise the performance and reliability of the Autonomous vehicle since sensors may find it difficult to detect the pavement markings especially in the work zone area where restriping, absence of hard surface and replaced with gravel and removed or obscured marking many times makes it very difficult for sensors, radar, LiDAR to detect them efficiently. Therefore, the work zone can present a variety of unusual circumstances not typically encountered during normal driving [23].

With AVs set to become reality from level 1 autonomous to level 5 autonomous stage. The efforts are being made to make its movement safe through the work zone and area of limitation. But due to the very dynamic nature of the work zone, it is very important to design new pavement marking which can be used exclusively for the work zone operations. Work zone usually has long term closure and short term closure. The current study is an effort to suggest as one of the ways which can be used for the safe movement of AVs through the work zone. The study also assumes that even though level 5 automation is achieved for AVs, there will still be a large percentage of human-driven vehicles on roads. Therefore, an autonomous vehicle will interact with human-driven vehicles while on roads. 
Since existing pavement markings and road infrastructure was never designed for the movement of AVs but only for the human drivers. Therefore, various issues like uneven work zone surface, confusing pavement striping, etc. were taken care of by human driver judgment and perception while driving through the work zone. Therefore, for the smooth movement of AVs through work zone new pavement markings have been designed as given in Table 1.

The pavement markings have been designed so that AVs can understand the lane closure and decide its path based on pavement markings. Figure 1 shows the detour plan for the South Bound and North Bound ramp closure for I-75. The designed pavement markings have been used as an example to show the possible movement using the new pavement markings. Figure 1 also shows the traffic control plans for human-driven vehicles as currently being used for any work zone during construction.

The pavement marking used to represent open lane has 6 inches white striping with 6 inches green striping pavement markings. The spacing between the white and green striping is 6 inches. Therefore, the total thickness of the pavement marking showing an open lane will be 30 inches. The thickness of pavement markings has been kept as 30 inches for all pavement markings mentioned in S. No. 1 to 9. The thickness for the work zone start and end pavement markings will have two 3 inch striping with 6 inches yellow and green/red striping will be 18 inches. A separate edge line striping running parallel throughout the work zone has been designed. This new striping will be used in place of a solid yellow edge line currently being used for highway pavement marking. The new edge line will have two 3 inches white striping with a solid 6-inch yellow line in the middle. The new edge line will have a thickness of 12 inches. Since the width of the edge line is now 12 inches in place of 6 inches solid yellow being used currently. The extra width will provide better detection by radar, LiDAR, and other sensors when AVs navigate through the work zone.

All the pavement markings will be placed throughout the width of the travel lane of 12 feet or less in case of reduced lane width. The lane open and lane closed pavement marking will be read by AVs for travel lane closure ahead. This can be used with specified distance marked along with pavement marking. For right turn single lane closure, the pavement marking has been split into two parts with right split showing red marking which will be detected by the AV as single-lane ramp closure on the right. The left split in the marking shown green middle striping with a white arrow which will be read by LiDAR as an open lane and move straight. Since AVs used High-resolution 3D maps and GPS along with radar and LiDAR and sensors. In Figure $2 \mathrm{SB}$ ramp "B" is closed. One AV reads the SB ramp " $\mathrm{B}$ " closure. It is instructed to move ahead. The GPS 3D maps will re-route the path of the AV and right turn sign for right ramp will guide it to make a right turn through ramp "B-1". The left split in this pavement marking using red middle striping will prevent the AVs to move ahead and make the right turn. 
Table 1. New pavement markings for movement of AV in work zone.

\begin{tabular}{|c|c|c|c|}
\hline No. & Marking Type & Pavement Marking & Pavement Configuration \\
\hline 1 & Lane Open & & $\begin{array}{c}6 " \text { White + 6" Green } \\
+6 " \text { Spacing }\end{array}$ \\
\hline 2 & Lane Closed & & $\begin{array}{l}\text { 6" White + 6" Green } \\
+6 " \text { Spacing }\end{array}$ \\
\hline 3 & $\begin{array}{l}\text { Right Turn Single Lane } \\
\text { Ramp Closure }\end{array}$ & & $\begin{array}{l}\text { 6" White + 6" Green } \\
+6 " \text { Spacing }\end{array}$ \\
\hline 4 & $\begin{array}{l}\text { Right Turn for Single Lane } \\
\text { right ramp closure }\end{array}$ & & $\begin{array}{l}\text { 6" White }+6 " \text { "Green }+ \\
6 " \text { Red }+6 " \text { Spacing }\end{array}$ \\
\hline 5 & Merge Right & & $\begin{array}{c}\text { 6" White }+6 \text { " Green }+ \\
6 " \text { Spacing }\end{array}$ \\
\hline 6 & $\begin{array}{l}\text { Left Turn Two-Lane } \\
\text { Ramp-Left Lane Closure }\end{array}$ & & $\begin{array}{l}\text { 6" White + 6" Green + } \\
\text { 6" Red + 6" Spacing }\end{array}$ \\
\hline 7 & $\begin{array}{c}\text { Left Turn Two-Lane } \\
\text { Ramp-Right Lane Closure }\end{array}$ & & $\begin{array}{c}6 " \text { White + 6" Green + 6" } \\
\text { Red + 6" Spacing }\end{array}$ \\
\hline 8 & $\begin{array}{l}\text { Left Turn Single Lane } \\
\text { Ramp Closure }\end{array}$ & & $\begin{array}{l}\text { 6" White + 6" Green + } \\
6 " \text { Red }+6 " \text { Spacing }\end{array}$ \\
\hline 9 & U-Turn & & $\begin{array}{c}\text { 6" White + 6" Green + 6" } \\
\text { Red + 6" Spacing }\end{array}$ \\
\hline 10 & Start Work Zone & & $\begin{array}{c}2 \times 3 " \text { White }+6 " \text { Yellow + } \\
6 " \text { Green }\end{array}$ \\
\hline 11 & End Work Zone & & $\begin{array}{c}2 \times 3 " \text { White }+6 " \text { Yellow }+ \\
6 " \text { Red }\end{array}$ \\
\hline 12 & $\begin{array}{c}\text { Work Zone Edge Line } \\
\text { (Contrast Pavement Marking) }\end{array}$ & & $2 \times 3 "$ White $+6 "$ Yellow \\
\hline
\end{tabular}

\subsection{New Pavement Markings for Autonomous Vehicles in the Work Zone}

New Pavement Markings for Movement of AV in Work Zone are listed in Table 1.

\subsection{Autonomous Vehicle Work Zone Movement: Example-I}

Figure 1 shows the detour plan for the SB and NB I-75 ramp closure. The figure shows the traffic control plans for the movement of human-driven vehicles. The 
same traffic control plan has been used in this study to show the movement of an autonomous vehicle using the dedicated pavement marking signs for its movement in the work zone. Figure 2 shows the movement autonomous vehicle after SB ramp "B" Closure. Figure 3 shows the movement of autonomous vehicles using new pavement marking when NB I-75 ramp "D-1" is closed. When the NB I-75 ramp "D-1 has closed the vehicle traveling to WB Laurel road cannot make a left turn to ramp "D-1" but will have to take a detour. The new detour plan for the autonomous vehicle will have work zone start sign for the WB traffic followed by the left turn single-lane ramp closure sign. The AVs will detect the sign and will proceed ahead. The AVs will move with the left edge lane marked for the construction. The AVs will now detect the U-turn sign which will have right split sign marked as red striping which directs AVs to make U-turn. As soon as AV makes the U-turn the AVs detect the marking which directs it to proceed and merge to the right lane so that it can merge with the ramp " $\mathrm{D}$ " to get on to NB I-75. Once the AV merges to its right it detects the right turn sign which directs it to get on to the ramp " $\mathrm{D}$ ". Once AV proceeds on-ramp " $\mathrm{D}$ " it will detect the end work zone sign. AV will follow the regular speed once the work zone ends.

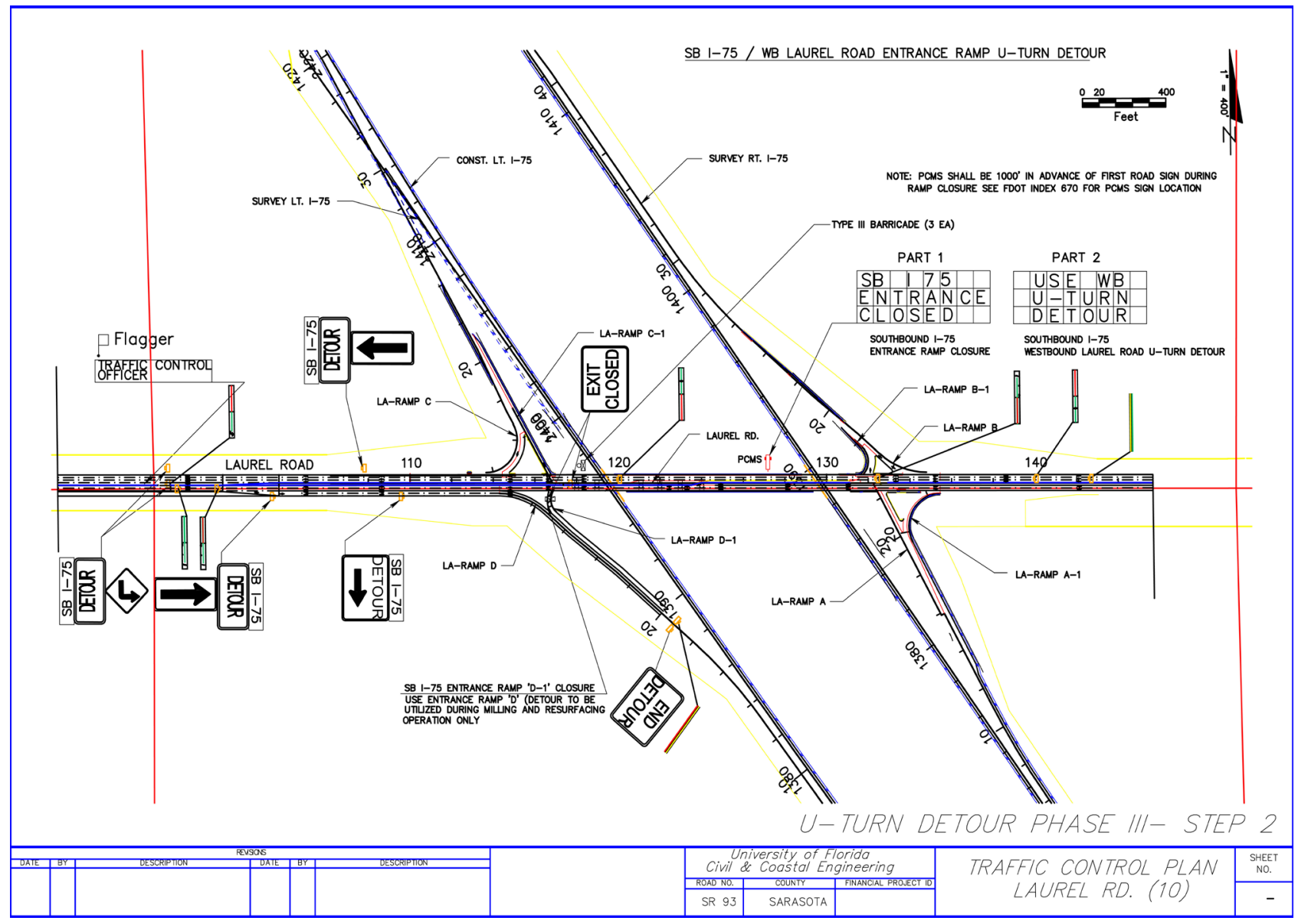

Figure 1. Detour Plan for NB and SB movement of AVs in work zone. 


\subsection{Autonomous Vehicle Work Zone Movement: Example-II}
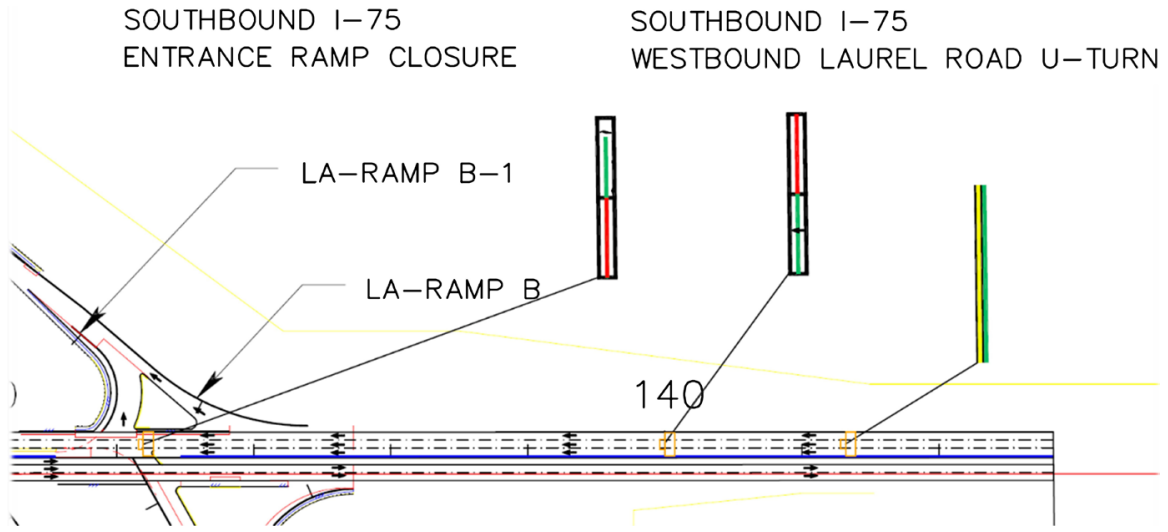

Figure 2. AV movement for SB Exit ramp "B" Closure in work zone.

\subsection{Autonomous Vehicle Work Zone Movement: Example-III}

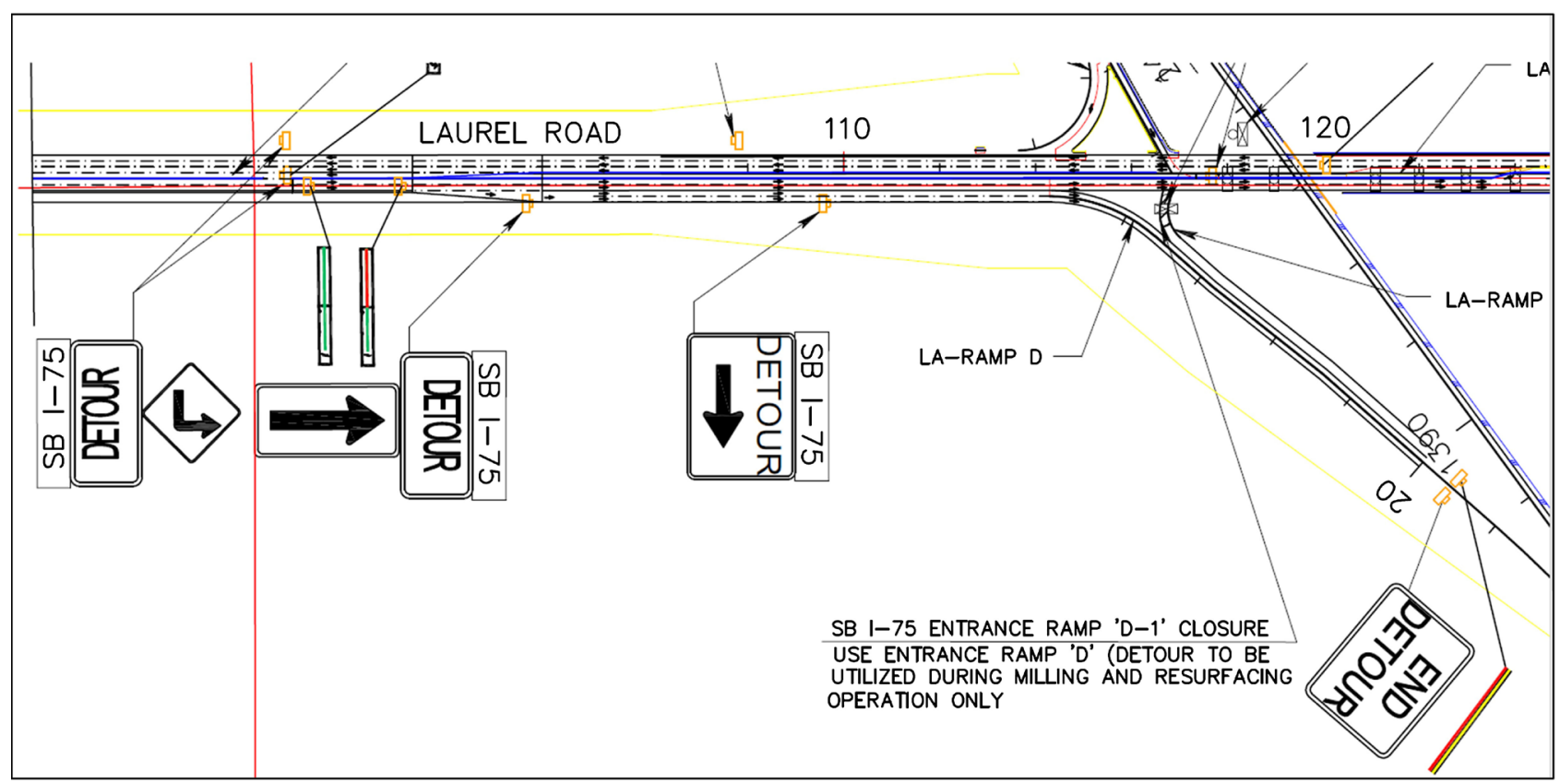

Figure 3. AV movement for NB ramp D-1 closure work zone.

\section{Using the Template}

Since the work zone usually has long term lane closure and short-term lane closure. The long-term lane closure could be from few days to few weeks but short-term lane closure could be from a few hours to a couple of days. For the short-term lane closure, it is important to guide the autonomous vehicle through complicated work zone since it is very difficult for the maps to get updated for short term lane closure. The study proposes to use raised pavement markers with a chip installed inside (Figure 4). This can use the available technologies like Surface Acoustic Wave Radio Frequency Identification tags (SAW RFID) or the installed chip can be detected by the onboard sensors by the autonomous vehicle. 


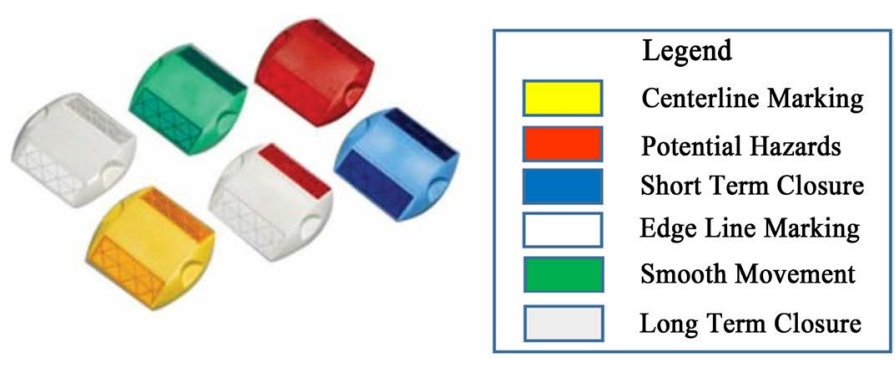

Figure 4. Raised pavement markers with the sensor chip.

The use of these technologies in raised pavement markers can be very useful for the smooth movement of an autonomous vehicle when used with a solid edge line. These markings can be installed very easily and are cost-effective especially when used for short term lane closure. Since winter weather hinder the performance of the LiDAR and other sensor devices especially when there is snow. This is because laser bouncing snowflakes is just one of many ways that AV can become befuddled by snow. SAW RFID, when used on raised pavement marking, can be one of the possible solutions since this can work in the snow. However, their performance needs to be tested with the movement of AVs in the work zone.

\section{Discussions}

As the results provided for a new set of pavement markings can be used as one of the options for the movement of AVs in the work zone. However, various issues need to be taken care of while writing an algorithm for the work zone. There are certain areas like autonomous vehicle making U-turn needs to be seen while deciding the placement of the pavement marking. In Figure 3 when AV makes a U-turn to merge on to ramp " $D$ ". The lane changing pattern of AVs needs to be considered with respect to traffic moving in EB direction. Apart from other issues linked with there is a need for developing a standard protocol for the autonomous vehicles moving in the work zone. There is also a need to develop standard traffic control plans and maintenance of traffic plans which can serve as a standard document for the work zone dealing with AVs.

\section{Conclusions}

AVs are set to revolutionize the transportation system and have a big expectation for a transportation engineer in improving mobility, level of service, cost-saving, safety. However, how the driving pattern changes in the future is still a question that will be answered in the future. Assumption of having any situation like increased use of AVs and complete stoppage of use of traditional vehicles is still very vague. Various factors will decide the use of AVs in the future as there could be more useful by 60 - 100 years age group rather than by the $20-60$ age group. Apart from this most of the research and simulation are based on the predictive data and each area of concern needs to be dealt with separately since the road infrastructure is dynamic and designed to be used by the human drivers. 
Though the world is excited about the future and everyone is looking to jump into the new transportation world of connected and AVs. But there are concerns and issues regarding the safety and reliability of the connected and AVs.

The autonomous vehicle will add cost not only to the manufacturing of cars and other AV vehicle but there will be a massive increase not only in the cost of infrastructure construction but maintenance and sustainability of infrastructure. The reason is the autonomous vehicle is expected to perform much better than the current human-driven vehicles. Their movement will not be restricted to any specific areas. This will put pressure to take infrastructure construction practice to the next level. New protocols and standards will be required to do the construction and move the vehicle in the work zone. This will not be limited to the work zone on interstate highways but also short term and long-term closure on minor streets, residential areas, parking lots, residential neighborhoods, etc.

Since most of the academic research is around the trajectory of the AVs and other aspects. Their interaction with the existing infrastructure and dynamic changes during construction and in an emergency is of importance and needs to be studied with. There is not much data available as to how AVs will respond to such a dynamic situation. Many efforts are being done by big companies like Google and Uber but their data is unavailable for research. The study put forth an idea that can be used to take it further and write a new set of algorithms that can be field-tested using an autonomous vehicle.

\section{Acknowledgements}

The authors express sincere gratitude to Dr. Siva Srinivasan, Associate Professor, Department of Civil and Coastal Engineering, the University of Florida for motivation and support in developing the concept.

\section{Conflicts of Interest}

The authors declare no conflicts of interest regarding the publication of this paper.

\section{References}

[1] Islam, M.A. (2018) Intergrading Connected Vehicle Data into the Transportation Performance Measurement Process. Doctoral Dissertation, The University of Alabama, Birmingham.

[2] Islam, M.A., Sisiopiku, V.P., Ramadan, O.E. and Hadi, M. (2019) A Framework for Performance-Based Traffic Operations Using Connected Vehicle Data. Simulation, 6, 10548-10555.

[3] Islam, M.A. (2017) Requirements and Challenges Associated with Deployment of Connected Vehicles.

[4] Qiao, F.X., Li, Q. and Yu, L. (2017) Intelligent Work Zone System and Connected Vehicle Technologies to Reduce Crashes in Roadway Construction Area. Civil Engineering Research Journal, 2, Issue 3, September 2017.

[5] Matowicki, M., Pribyl, O. and Pribyl, P. (2016) Smart City Symposium Prague. 
Analysis of Possibility to Utilize Road Marking for the Need of Autonomous Vehicle. Smart Cities Symposium, Prague, 26-27 May 2016.

https://doi.org/10.1109/SCSP.2016.7501026

[6] Toro, O., Becsi, T. and Aradil, S. (2016) Design of Lane Keeping Algorithm of Autonomous Vehicle. Periodica Polytechnica Transportation Engineering, 44, 60-68. https://doi.org/10.3311/PPtr.8177

[7] Dickmanns, E.D. (2001) The Development of Machine Vision for Road Vehicles in Last Decade. Proc. Intelligent Vehicle, Vol. 1, 268-281.

[8] Duchow, C. (2005) A Marking-Based, Flexible Approach to Intersection Detection. IEEE Computer Society Conference on Computer Vision and Pattern Recognition, San Diego, 20-26 June 2005.

[9] Enkelmann, W., Struck, G. and Geisler, J. (1995) ROMA-A System for Model-Based Analysis of Road Markings. IEEE Intelligent Vehicles Symposium, Detroit, MI, 25-26 September 1995, 356-360.

[10] Anderson, J.M., Kalra, N., Stanley, K.D., Sorensen, P., Samaras, C. and Oluwatola, O.A. (2016) Autonomous Vehicle Technology: A Guide for Policymakers. Rand Corporation, Santa Monica. https://doi.org/10.7249/RR443-2

[11] Vacek, S., Schimmel, C. and Dillman, R. (2007) Road Marking Analysis for Autonomous Vehicle Guidance. Proceedings of the 3rd European Conference on Mobile Robots, Freiburg, 19-21 September 2007.

[12] Voronov, A., Hulten, J., Wedlin, J. and England, C. (2016) Radar Reflecting Pavement Markers for Vehicle Automation.

[13] Narote, S.P., Bhujbal, P.N., Narote, A.S. and Dhane, D.M. (2017) A Review of Recent Advances in Lane Detection and Departure Warning System. Pattern Recognition, 73, 216-234. https://doi.org/10.1016/j.patcog.2017.08.014

[14] Hernandez, D.C., Kumiabggoro, L., Filonenko, A. and Jo, K.H. (2016) Real Time Lane Region Detection Using a Combination of Geometrical and Image Feature. Sensors, 16, 1935. https://doi.org/10.3390/s16111935

[15] Mekker, M.M., Lin, Y.-J., Elbahnasawy, M.K.L., Shamseldin, T.S.A., Li, H., Habib, A.F. and Bullock, D.M. (2018) Application of Lidar and Connected Vehicle Data to Evaluate the Impact of Work Zone Geometry on Freeway Traffic Operation. Transportation Research Record, 2672, 1-13. https://doi.org/10.1177/0361198118758050

[16] US Department of Transportation, Federal Highway Administration (2015) Use of Radio Frequency Tags in Pavements. No. FHWA-HRT-14-061.

[17] Bryden, J.E. and Andrew, L.B. (2001) Quality Assurance Program for Work Zone Traffic Control. Transportation Research Record: Journal of the Transportation Research Board, 1745, 1-9. https://doi.org/10.3141/1745-01

[18] Andrew, L.B. and Bryden, J.E. (1997) Managing Construction Safety and Health: Experience of New York State Department of Transportation. Transportation Research Record: Journal of the Transportation Research Board, 1585, 9-18. https://doi.org/10.3141/1585-02

[19] Singh, P. (2005) A Study of Fatal Run off Road Crashes in the State of Florida. FSU-MS Thesis.

[20] Prashant, S. (2004) Safety Analysis of Fatal Crashes Involving High Speed Vehicles. International Traffic Record Forum, Nashville.

https://2004.trafficrecordsforum.org/Sessions/Wednesday_25-36/S36/s36_trf_paper singh_and_spainhour.pdf 
[21] Islam, M.A. (2019) A Literature Review on Freeway Traffic Incidents and Their Impact on Traffic Operations. Journal of Transportation Technologies, 9, 504-516. https://doi.org/10.4236/jtts.2019.94032

[22] Terry, T.N., Tanner, S., National Surface Transportation Safety Center for Excellence (NSTSCE) (2018) Problematic Roadway Environments for Automated Vehicles.

[23] Ieng, S.-S. and Labayrade, J.-P.T.R. (2003) On the Design of a Single Lane-Markings Detector Regardless the On-Board Camera's Position. IEEE Intelligent Vehicles Symposium, Columbus, 9-11 June 2003, 564-569. 